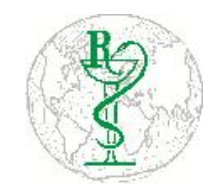

INDO GLOBAL JOURNAL OF

PHARMACEUTICAL SCIENCES

ISSN 2249- 1023

\title{
Synthesis, Urease Inhibition, Antimicrobial and Antioxidant Studies of 3,4,5-Trihydroxy Benzoic Acid Derivatives
}

\author{
Kalyan Mondal, Shashi Sharma, Saloni Kakkar " , Anurag Khatkar \\ Department of Pharmaceutical Sciences, Maharshi Dayanand University, Rohtak-124001, India
}

\begin{abstract}
Address for
Correspondance

Saloni Kakkar, $\underline{\text { salonikakkar2007 }}$

@ gmail.com
\end{abstract}

Received:

23.02.2017

Accepted:

30.04.2017

\begin{abstract}
A series of fourteen 3, 4, 5-trihydroxy benzoic acid were synthesized by schematic route shown in scheme 1 and characterized by spectroscopic analysis. All the newly synthesized derivatives were evaluated for their antimicrobial activity against Gram-positive bacteria: Staphylococcus aureus, Bacillus subtilis, Gram-negative bacteria: Escherichia coli, and fungal strains: Candida albicans and Aspergillus niger by tube dilution method. The compounds were also evaluated for antioxidant potential using DPPH free radical assay. Results of antimicrobial screening indicated that compound $1\left(\mathrm{MIC}_{\mathrm{CA}}=\mathrm{MIC}_{\mathrm{AN}}=0.10 \mu \mathrm{M} / \mathrm{ml}\right)$ was found to be most active antimicrobial agent comparable to standard drugs taken as well as showed good antioxidant potential $\left(\mathrm{IC}_{50}=49.38 \mu \mathrm{g} / \mathrm{ml}\right)$. The synthesized derivatives $(\mathbf{1 - 1 4})$ were evaluated in vitro for their urease inhibitory activities by Indophenol method; most of the investigated compounds were potent inhibitors of Jack Bean Urease. The compounds 13 and 1 were found to be more potent urease inhibitors than the standard thiourea, depicting the $\mathrm{IC}_{50}$ values of 0.07 and $0.08 \mu \mathrm{M} / \mathrm{ml}$, respectively $\left(\mathrm{IC}_{50}\right.$ of thiourea $=0.61$ $\mu \mathrm{M} / \mathrm{ml})$. (C) 2018 iGlobal Research and Publishing Foundation. All rights reserved.
\end{abstract}

Keywords Antimicrobial; Antioxidant; Urease Inhibition; 3,4,5-Trihydroxy Benzoic Acid Derivatives.

\section{INTRODUCTION}

Over the last era, the number of antibiotic-resistant Grampositive and Gram-negative bacteria is reaching its crucial point around the world. [1] The growing prevalence of multidrug resistant strains of bacteria and the current appearance of new strains with decreased susceptibility to antibiotics raises the specter of untreatable bacterial infections.

[2] Therefore, there is an urgent need to develop anti-infective agents for the treatment of bacterial diseases. Recently, the research has been focused toward development of new antibacterial agents which may result in the discovery of novel effective compounds. [3] The limitations of current antifungal drugs, increased incidence of systemic fungal infections, and rapid development of drug resistance have highlighted the need for the discovery of new antifungal agents, preferably with novel mechanisms of action. [4]
Urease (urea amidohydrolase; E.C. 3.5.1.5) is a nickel containing metalloenzyme that rapidly catalyzes the hydrolysis of urea to the formation of ammonia and carbon dioxide. [5] It plays an important role in nitrogen metabolism of plants during the germination process. [6] A variety of ureases have been isolated from algae, bacteria, fungi, and plants. [5-7, 8] Irrespective of structural differences of plant and microbial originated urease, it follows same catalysis pattern. It is mainly because of similar sequence of amino acids and presence of $\mathrm{Ni}^{+2}$ ions in active site of this multimeric enzyme which indicates emergence from a common ancestry. [5, 10, 11]

The main physiological role of urease is to provide nitrogen for organisms in the form of ammonia for their growth. However, high urease activity is responsible for release of abnormally large amounts of ammonia into atmosphere which may lead to environmental and economic problems. [5, 6] 
Indo Global Journal of Pharmaceutical Sciences, 2018; 8(1): 12-20

Urease producing bacteria have a harmful effect on human health. In humans the urease of Helicobacter pylori causes infections of urinary and gastrointestinal tract [7], such as peptic ulcer and the stomach cancer. [12] Other urease associated diseases include hepatic encephalopathy, urolithiasis, urinary catheter encrustation, pyelonephritis and hepatic coma. [5] The urease activity of Helicobacter pylori plays an important role in the pathogenesis of peptic and gastric ulcer. [12] Therefore, urease inhibitors have the potential to be used as anti-ulcer drugs. Ureases are inhibited by different classes of compounds. Thiourea and their derivatives were found to have a significant inhibitory activity against the urease enzyme. Thiourea attracted worldwide attention due to their number of applications and diverse biological significance, possess a wide range of biological applications including antiviral, antibacterial, antifungal, antitubercular, antithyroid, herbicidal, insecticidal activities and they act as agrochemicals [9].

In this paper, we present the synthesis of 3, 4, 5-trihydroxy benzoic acid derivatives having wide range of biological activities and their evaluation for inhibitory activity against Jack bean urease. It is notable that most of the compounds were more potent inhibitors of the enzyme as compared to standard inhibitor (thiourea). One of the compounds (13) had most potent urease inhibitory activity with $\mathrm{IC}_{50}$ value of $0.07 \mathrm{M} / \mathrm{ml}$, which is more active than the standard (0.61

$\mathrm{M} / \mathrm{ml}$ ). Newly synthesized compounds were also investigated on pathogenic microbial strains and it was observed that most of the compounds also exhibited potent antimicrobial activities.

\section{MATERIALS AND METHODS}

All reagents and solvents used in study were of analytical grade and procured locally. Melting points were determined in open capillary tubes on a Sonar melting point apparatus and are uncorrected. The progress of the reaction was monitored by thin layer chromatography on silica gel coated glass plate and products were purified through recrystallization and purity of the compounds was checked by thin layer chromatography (TLC) performed on silica gel G coated plates in the solvent system Benzene : Chloroform (7:3), and the spots were located by iodine. $\mathrm{H}^{1}$ nuclear magnetic resonance $\left(\mathrm{H}^{1} \mathrm{NMR}\right)$ spectra were recorded on Agilent NMR $300.13100000 \mathrm{MHz}$ spectrometer using DMSO solvent and are expressed in parts per million $(\delta, \mathrm{ppm})$. Infra-red (IR) spectra were recorded on Brucker 12060280, Software: OPUS 7.2.1394 spectrophotometer.
1. General procedure for synthesis of 3,4,5trihydroxybenzoyl chloride

Thionyl chloride $(0.6 \mathrm{~mol})$ was added gently to gallic acid $(0.35 \mathrm{~mol})$ in a round bottom flask. After completion of the addition of thionyl chloride, the mixture was stirred for $4 \mathrm{~h}$ and then heated to $80^{\circ} \mathrm{C}$ for 30 minutes in water bath. The end point of the reaction was detected by using thin layer chromatography. After the completion of the reaction, solid mass was filtered and washed with toluene to obtain 3,4,5trihydroxybenzoyl chloride.

2. General procedure for synthesis of quinolin-8-yl 3,4,5trihydroxybenzoate

The solution of 8-hydroxy quinolin $(0.025 \mathrm{~mol})$ in ether $(15$ $\mathrm{mL}$ ) was added to a solution of 3,4,5-trihydroxybenzoyl chloride $(0.025 \mathrm{~mol})$ in ether $(15 \mathrm{~mL})$. Then, the mixture was heated for 45 minutes in water bath. The reaction monitoring was done by using TLC. The mixture was cooled at room temperature and evaporation of solvent yielded the crude ester which was purified by recrystallization with alcohol.

\section{General procedure for synthesis of amides}

The solution of corresponding aniline $(0.025 \mathrm{~mol})$ in ether $(15$ $\mathrm{mL})$ was added drop wise to a solution of 3,4,5trihydroxybenzoyl chloride $(0.025 \mathrm{~mol})$ in ether $(15 \mathrm{~mL})$ with temperature maintained between at $0-10{ }^{\circ} \mathrm{C}$ (Scheme 1). The solution was stirred for $45 \mathrm{~min}$ and the precipitated amide was separated out. The crude amide was recrystallized with alcohol.

\section{In vitro antimicrobial activity}

The antimicrobial activity of synthesized compounds (1-14) was tested against Gram-positive bacteria: Staphylococcus aureus MTCC 3160, Bacillus subtilis MTCC 441, Gramnegative bacterium: Escherichia coli MTCC 443 and fungal strains: Candida albicans MTCC 227and Aspergillus niger MTCC 281 using the tube dilution method. Fluconazole (antifungal) and norfloxacin (antibacterial) were used as reference drugs [6]. Dilutions of the test and standard compounds were prepared in double strength nutrient broth I.P. (bacteria) or Sabouraud dextrose broth I.P. (fungi) [7]. The samples were incubated at $37 \pm 1^{\circ} \mathrm{C}$ for $24 \mathrm{~h}$ (bacteria), at $37 \pm$ $1^{\circ} \mathrm{C}$ for $48 \mathrm{~h}$ (C. albicans) and at $25 \pm 1^{\circ} \mathrm{C}$ for 7 days (A. niger) respectively and the results were recorded in terms of MIC (the lowest concentration of test substance which inhibited the growth of microorganisms). [8, 9]

\section{In vitro antioxidant activity}

The antioxidant activity of the synthesized compounds (1-14) and the standard (ascorbic acid) was assessed on the basis of the radical scavenging effect of the stable 1, 1-diphenyl-2picrylhydrazyl (DPPH)-free radical activity. The 
Indo Global Journal of Pharmaceutical Sciences, 2018; 8(1): 12-20

spectrophotometric assay uses the stable radical DPPH as a reagent fifty milliliters of various concentrations of the compounds dissolved in methanol was added to $5 \mathrm{~mL}$ of a $0.004 \%$ methanol solution of DPPH. After a $30 \mathrm{~min}$ incubation period at room temperature, the absorbance was read against a blank at $517 \mathrm{~nm}[9,10]$. Compounds concentration providing $50 \%$ inhibition $\left(\mathrm{IC}_{50}\right)$ was calculated from the graph plotted as inhibition percentage against compound concentration. [10]

\section{In vitro Urease Inhibitory Activity}

Urease inhibitory activity was assessed for all synthesized compounds using Jack Bean Urease by Indophenol method [5]. In brief, $40 \mathrm{~L}$ buffer (100 mM urea, $1 \mathrm{mM}$ EDTA, $0.01 \mathrm{M}$ $\mathrm{K}_{2} \mathrm{HPO}_{4}, 0.01 \mathrm{M} \mathrm{LiCl}_{2}, \mathrm{pH} 8.2$ ), $10 \mathrm{~L}$ of test compound and $10 \mathrm{~L}$ of enzyme $(5 \mathrm{U} / \mathrm{mL})$ solution were incubated at $37^{\circ} \mathrm{C}$ for $10 \mathrm{~min}$. After completion of the addition, solutions of $40 \mathrm{~L}$ of alkali reagent $(0.5 \%$, w/v $\mathrm{NaOH}, 0.1 \%$ active chloride $\mathrm{NaOCl})$ and $40 \mathrm{~L}$ of phenol reagent $(1 \%$, w/v phenol, $0.005 \%$, w/v sodium nitroprusside) were introduced to each well. Experiment study was determined at $625 \mathrm{~nm}$ in UV spectrophotometer and thiourea was used as standard inhibitor.

\section{RESULTS AND DISCUSSION}

\section{Chemistry}

Synthesis of compounds (1-14) followed the general pathway outlined in the Scheme 1. All the gallic acid derivatives were synthesized in good yield. The physicochemical properties of the synthesized compounds are presented in Table 2. The structures of all the newly synthesized compounds were confirmed by the IR, and ${ }^{1} \mathrm{H}$ NMR which were in full agreement with their structures.

Scheme 1: Scheme for the synthesis of 3, 4, 5-trihydroxy benzoic acid derivatives
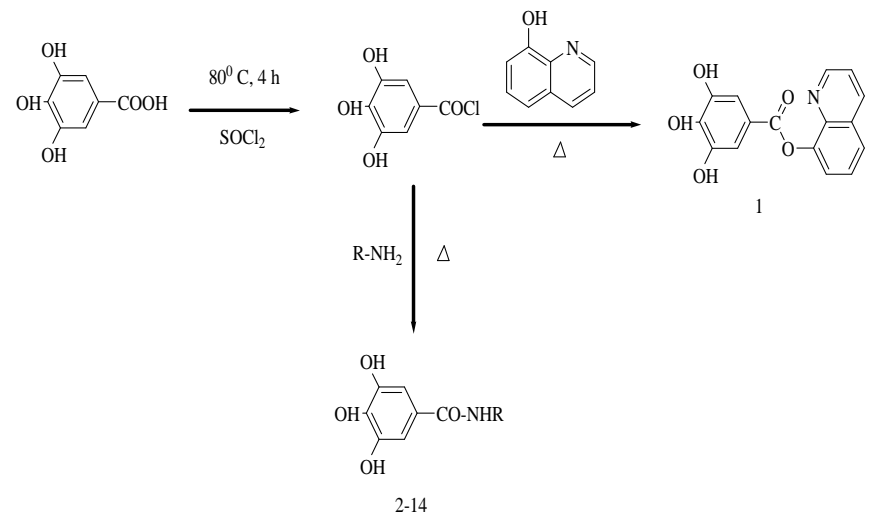

\section{SPECTRAL DATA:}

3,4,5-trihydroxybenzoyl chloride (Intermediates)

IR (KBr pellets) $\mathrm{cm}^{-1}: 3495(\mathrm{O}-\mathrm{H}$ str., phenol), 3066(C-H str., phenyl nucleus), 1541 ( $\mathrm{C}=\mathrm{C}$ skeletal str., phenyl), $733(\mathrm{C}-\mathrm{Cl}$ str., aromatic); ${ }^{1} \mathbf{H}$ NMR (DMSO-d 6 , $\mathbf{\delta p p m ) : ~} 6.72$ (d, 2H, Ar$\mathrm{H})$.

Quinolin-8-yl 3,4,5-trihydroxy benzoate (1)

IR (KBr pellets) $\mathrm{cm}^{-1}$ : 3445 (O-H str., phenol), 3098 (C-H str., aromatic), 1505 ( $\mathrm{C}=\mathrm{C}$ skeletal str., phenyl), 1354 (ring str., quinoline), 1225 (C-O str., ester); ${ }^{1} \mathbf{H}$ NMR (DMSO-d 6 , oppm): 7.02 (d, 2H,Ar-H), 6.95-8.89 (m, 6H, qunoline)

\section{3,4,5-trihydroxy-N-phenyl benzamide (2)}

IR (KBr pellets) $\mathrm{cm}^{-1}: 3345$ (O-H str., phenol), 3228 (N-H str., $2^{\circ}$ amide), 3097 (C-H str., aromatic), 1583 (N-H bending, $2^{\circ}$ amide), 1583 ( $\mathrm{C}=\mathrm{C}$ skeletal str., phenyl); ${ }^{\mathbf{1}} \mathbf{H}$ NMR (DMSO-

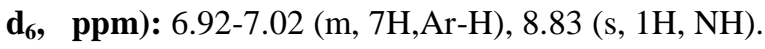

\section{$N$-(2-chlorophenyl)-3,4,5-trihydroxy benzamide (3)}

IR ( $\mathrm{KBr}$ pellets) $\mathrm{cm}^{-1}: 3496$ (O-H str., phenol), 3281 (N-H str., $2^{\circ}$ amide), $1665\left(\mathrm{C}=\mathrm{O}\right.$ str., $2^{\circ}$ amide), 1611 ( $\mathrm{N}-\mathrm{H}$ bending, $2^{\circ}$ amide), 1539 (C=C skeletal str., phenyl), 733 (C-Cl str., aromatic) ${ }^{1}{ }^{\mathbf{H}}$ NMR (DMSO-d $\left.{ }_{\mathbf{6}}, \mathbf{\delta p p m}\right):$ 6.84-8.61 (m, 6H,Ar$\mathrm{H}), 8.61(\mathrm{~s}, 1 \mathrm{H}, \mathrm{NH})$

\section{3,4,5-trihydroxy-N-(2-nitrophenyl)benzamide(4)}

IR (KBr pellets) $\mathrm{cm}^{-1}: 3493$ (O-H str., phenol), 3350 (N-H str., $2^{\circ}$ amide), $1616\left(\mathrm{C}=\mathrm{O}\right.$ str., $2^{\circ}$ amide), $1616\left(\mathrm{~N}-\mathrm{H}\right.$ bending, $2^{\circ}$ amide), 1569 (C=C skeletal str., phenyl), 1385 ( $\mathrm{NO}_{2}$ sym. str.,

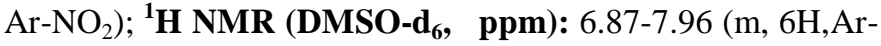
H), 7.96 (s, 1H, NH).

\section{3,4,5-trihydroxy-N-(3-nitrophenyl)benzamide(5)}

IR (KBr pellets) $\mathrm{cm}^{-1}: 3495$ (O-H str., phenol), 3431 (N-H str., $2^{\circ}$ amide), 3073 (C-H str., aromatic ring), 1520 (C=C skeletal str., phenyl), 1618 ( $\mathrm{C}=\mathrm{O}$ str., $2^{\circ}$ amide), 1618 (N-H bending, $2^{\circ}$ amide), $1384\left(\mathrm{NO}_{2}\right.$ sym. str., $\left.\mathrm{Ar}-\mathrm{NO}_{2}\right){ }^{1} \mathbf{H}$ NMR (DMSO-

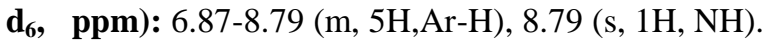

\section{$N$-(2,4-dinitrophenyl)-3,4,5-trihydroxy benzamide (6)}

IR (KBr pellets) cm $\mathrm{cm}^{-1}: 3495$ (O-H str., phenol), 3337 (N-H str., $2^{\circ}$ amide), 3107 (C-H str., aromatic ring), 1584 ( $\mathrm{C}=\mathrm{C}$ skeletal str., phenyl), 1628 ( $\mathrm{C}=\mathrm{O}$ str., $2^{\circ}$ amide and $\mathrm{N}-\mathrm{H}$ bending, $2^{\circ}$ amide), 1332 ( $\mathrm{NO}_{2}$ sym. str., $\left.\mathrm{Ar}-\mathrm{NO}_{2}\right) ;{ }^{\mathbf{1}} \mathbf{H}$ NMR (DMSO-d 6 , סppm): 6.86-9.16 (m, 5H,Ar-H), 8.13 (s, 1H, NH).

$N$-(4-chlorophenyl)-3,4,5-trihydroxy benzamide (7)

IR (KBr pellets) $\mathrm{cm}^{-1}: 3459$ (O-H str., phenol), 3459 (N-H str., $2^{\circ}$ amide), 3081 (C-H str., aromatic ring), 1624 (N-H bending, $2^{\circ}$ amide), 1547 ( $\mathrm{C}=$ Cskeletal str., phenyl), 1653 ( $\mathrm{C}=\mathrm{O}$ str., $2^{\circ}$ amide), 733 (C-Cl str., aromatic); ${ }^{1} \mathbf{H}$ NMR (DMSO-d 6 , бppm): 6.78-6.99 (m, 6H,Ar-H), 8.56 (s, 1H, NH). 
Indo Global Journal of Pharmaceutical Sciences, 2018; 8(1): 12-20

Table 1: Arrangement of substituent's used for targeted compounds

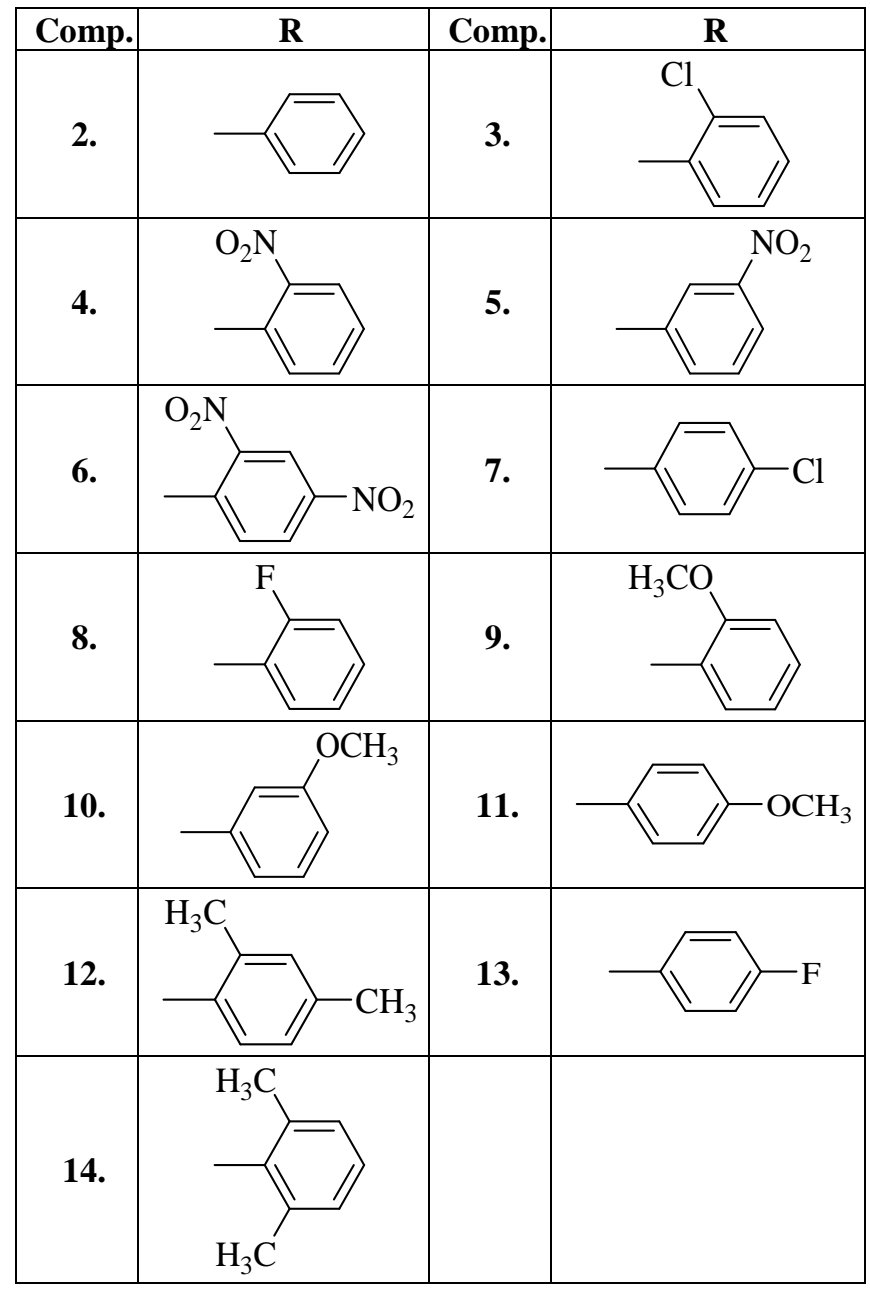

Table 2: Physicochemical properties of synthesized 3, 4, 5-trihydroxy benzoic acid derivatives (1-14).

\begin{tabular}{|c|c|c|c|c|c|}
\hline Comp. & Mol. Formula & M. Wt. & m.p. ( $\left.{ }^{\mathbf{0}} \mathbf{C}\right)$ & Rf Value* & \% Yield \\
\hline $\mathbf{1 .}$ & $\mathrm{C}_{16} \mathrm{H}_{11} \mathrm{NO}_{5}$ & 297.26 & $195-205$ & 0.54 & 38.88 \\
\hline $\mathbf{2 .}$ & $\mathrm{C}_{13} \mathrm{H}_{11} \mathrm{NO}_{4}$ & 245.23 & $170-180$ & 0.33 & 88.23 \\
\hline $\mathbf{3 .}$ & $\mathrm{C}_{13} \mathrm{H}_{10} \mathrm{ClNO}_{4}$ & 279.68 & $180-185$ & 0.68 & 93.33 \\
\hline $\mathbf{4 .}$ & $\mathrm{C}_{13} \mathrm{H}_{10} \mathrm{~N}_{2} \mathrm{O}_{6}$ & 290.23 & $140-145$ & 0.54 & 82.35 \\
\hline $\mathbf{5 .}$ & $\mathrm{C}_{13} \mathrm{H}_{10} \mathrm{~N}_{2} \mathrm{O}_{6}$ & 290.23 & $137-140$ & 0.41 & 73.33 \\
\hline $\mathbf{6 .}$ & $\mathrm{C}_{13} \mathrm{H}_{9} \mathrm{~N}_{3} \mathrm{O}_{8}$ & 335.23 & $130-135$ & 0.60 & 70.00 \\
\hline $\mathbf{7 .}$ & $\mathrm{C}_{13} \mathrm{H}_{10} \mathrm{ClNO}_{4}$ & 279.68 & $190-193$ & 0.46 & 40.00 \\
\hline $\mathbf{8 .}$ & $\mathrm{C}_{13} \mathrm{H}_{10} \mathrm{FNO}_{4}$ & 263.22 & $148-152$ & 0.32 & 40.00 \\
\hline $\mathbf{9 .}$ & $\mathrm{C}_{14} \mathrm{H}_{13} \mathrm{NO}_{5}$ & 275.26 & $158-162$ & 0.50 & 22.85 \\
\hline $\mathbf{1 0 .}$ & $\mathrm{C}_{14} \mathrm{H}_{13} \mathrm{NO}_{5}$ & 275.26 & $100-105$ & 0.27 & 93.22 \\
\hline $\mathbf{1 1 .}$ & $\mathrm{C}_{14} \mathrm{H}_{13} \mathrm{NO}_{5}$ & 275.26 & $102-105$ & 0.50 & 80.00 \\
\hline $\mathbf{1 2 .}$ & $\mathrm{C}_{15} \mathrm{H}_{15} \mathrm{NO}_{4}$ & 273.28 & $103-106$ & 0.33 & 82.00 \\
\hline $\mathbf{1 3 .}$ & $\mathrm{C}_{13} \mathrm{H}_{10} \mathrm{FNO}_{4}$ & 263.22 & $180-185$ & 0.68 & 91.67 \\
\hline $\mathbf{1 4 .}$ & $\mathrm{C}_{15} \mathrm{H}_{15} \mathrm{NO}_{4}$ & 273.28 & $162-167$ & 0.67 & 22.22 \\
\hline
\end{tabular}

*TLC mobile phase: - Chloroform: Benzene (3:7) 


\section{Indo Global Journal of Pharmaceutical Sciences, 2018; 8(1): 12-20}

$N$-(2-fluorophenyl)-3,4,5-trihydroxy benzamide (8)

IR (KBr pellets) $\mathrm{cm}^{-1}: 3282$ (O-H str., phenol and N-H str., $2^{\circ}$ amide), 3064 (C-H str., aromatic ring), $1700\left(\mathrm{C}=\mathrm{O}\right.$ str., $2^{\circ}$ amide), 1618 (N-H bending, $2^{\circ}$ amide), $1539(\mathrm{C}=\mathrm{C}$ skeletal str., phenyl), 1099 (C-F str., aromatic); ${ }^{1}$ H NMR (DMSO-d ${ }_{6}$, סppm): 6.81-7.63 (m, 6H,Ar-H), 7.95 (s, 1H, NH).

\section{3,4,5-trihydroxy-N-(2-methoxyphenyl)benzamide(9)}

IR (KBr pellets) $\mathrm{cm}^{-1}: 3495$ (O-H str., phenol), 3277 (N-H str., $2^{\circ}$ amide), $1608(\mathrm{C}=\mathrm{C}$ skeletal str., phenyl and $\mathrm{N}-\mathrm{H}$ bending, $2^{\circ}$ amide), 1315 (C-O-C str., $\mathrm{Ar}-\mathrm{OCH}_{3}$ );

\section{3,4,5-trihydroxy- $N$-(3-methoxyphenyl)benzamide(10)}

IR (KBr pellets) $\mathrm{cm}^{-1}: 3463$ (O-H str., phenol), 3345 (N-H str., $2^{\circ}$ amide), 1597 ( $\mathrm{N}-\mathrm{H}$ bending, $2^{\circ}$ amide), 1495 ( $\mathrm{C}=\mathrm{C}$ skeletal str., phenyl), 1263 (C-O-C str., Ar-OCH ${ }_{3}$ ); ${ }^{1}$ H NMR (DMSO$\left.\mathbf{d}_{\mathbf{6}}, \mathbf{\delta p p m}\right)$ : 6.86-7.20 (m, 6H,Ar-H), 8.85 (s, 1H, NH), 3.75 (t, $3 \mathrm{H}, \mathrm{OCH}_{3}$ ).

\section{3,4,5-trihydroxy- $N$-(4-methoxyphenyl)benzamide(11)}

IR (KBr pellets) $\mathrm{cm}^{-1}: 3382$ (O-H str., phenol), $3382(\mathrm{~N}-\mathrm{H}$ str., $2^{\circ}$ amide), 3006 (C-H str., aromatic ring), 1593 (C=C skeletal str., phenyl and $\mathrm{N}-\mathrm{H}$ bending, $2^{\circ}$ amide), 1300 (C-O-C str., $\mathrm{Ar}-\mathrm{OCH}_{3}$ ); ${ }^{1} \mathbf{H}$ NMR (DMSO-d $\left.\mathbf{6}, \mathbf{\delta p p m}\right)$ : 6.77-7.38 (m, $6 \mathrm{H}, \mathrm{Ar}-\mathrm{H}), 7.38(\mathrm{~s}, 1 \mathrm{H}, \mathrm{NH}), 3.74\left(\mathrm{t}, 3 \mathrm{H}, \mathrm{OCH}_{3}\right)$.

\section{$N$-(2,4-dimethylphenyl)-3,4,5-trihydroxy benzamide (12)}

IR (KBr pellets) $\mathrm{cm}^{-1}: 3353$ (O-H str., phenol), 3227 (N-H str., $2^{\circ}$ amide $), 2831\left(\mathrm{Ar}-\mathrm{CH}_{3}\right), 1629\left(\mathrm{C}=\mathrm{O}\right.$ str., $2^{\circ}$ amide and $\mathrm{N}-\mathrm{H}$ bending, $2^{\circ}$ amide), 1588 ( $\mathrm{C}=\mathrm{C}$ skeletal str., phenyl), ${ }^{1} \mathbf{H}$ NMR (DMSO-d $\left.{ }_{6}, \mathbf{\delta p p m}\right):$ 6.78-7.02 (m, 5H,Ar-H), $7.96(\mathrm{~s}, 1 \mathrm{H}$, $\mathrm{NH}), 2.50\left(\mathrm{~m}, 6 \mathrm{H}, \mathrm{CH}_{3}\right)$.

\section{$\mathrm{N}$-(4-fluorophenyl)-3,4,5-trihydroxy benzamide (13)}

IR ( $\mathrm{KBr}$ pellets) $\mathrm{cm}^{-1}$ : 3202 (O-H str., phenol and N-H str., $2^{\circ}$ amide),3111 (C-H str., aromatic ring), 1592 (C=C skeletal str., phenyl), $1629\left(\mathrm{C}=\mathrm{O}\right.$ str., $2^{\circ}$ amide and $\mathrm{N}-\mathrm{H}$ bending, $2^{\circ}$ amide), 1095 (C-F str., aromatic); ${ }^{1} \mathbf{H}$ NMR (DMSO-d 6 , סppm): 6.81-7.81(m, 6H,Ar-H), 7.85 (s, 1H, NH).

\section{$N$-(2,6-dimethylphenyl)-3,4,5-trihydroxy benzamide (14)}

IR (KBr pellets) $\mathrm{cm}^{-1}: 3495$ (O-H str., phenol), 3280 (N-H str., $2^{\circ}$ amide), 3064 (C-H str., aromatic ring), 1541 ( $\mathrm{C}=\mathrm{C}$ str., skeletal str., phenyl), 1609 ( $\mathrm{C}=\mathrm{O}$ str., $2^{\circ}$ amide and $\mathrm{N}-\mathrm{H}$ bending, $2^{\circ}$ amide); ${ }^{1} \mathbf{H}$ NMR (DMSO-d $\left.{ }_{\mathbf{6}}, \mathbf{\delta p p m}\right):$ 6.77-6.97 $(\mathrm{m}, 5 \mathrm{H}, \mathrm{Ar}-\mathrm{H}), 8.60(\mathrm{~s}, 1 \mathrm{H}, \mathrm{NH}), 2.36\left(\mathrm{~m}, 6 \mathrm{H}, \mathrm{CH}_{3}\right)$.

\section{In vitro antimicrobial activity}

All the newly synthesized compounds (1-14) were investigated for their in vitro antimicrobial activity against different Gram positive bacteria (B. subtilis and S. aureus),
Gram negative bacterium (E. coli) and fungal strains $(C$. albicans and A. niger) by the tube dilution method $[6,7,8]$ and the results of antimicrobial screening are presented in Table 3. Compound $\mathbf{1}$ was showed very potent antifungal activity $\left(\mathrm{MIC}_{\mathrm{CA}}=\mathrm{MIC}_{\mathrm{AN}}=0.10 \mu \mathrm{M} / \mathrm{ml}\right)$ against both the fungal strains as well as moderately active antibacterial activity $\left(\mathrm{MIC}_{\mathrm{EC}, \mathrm{BS}, \mathrm{SA}}=0.42 \mu \mathrm{M} / \mathrm{ml}\right)$ against all the three strains of bacteria and were comparable with the standard drugs taken. From the observed results, compounds 6 was found to be most active against $E$. coli $\left(\mathrm{MIC}_{\mathrm{EC}}=0.37 \mu \mathrm{M} / \mathrm{ml}\right)$ and also compounds 1,3 and 13 were found to be moderately active against $E$. coli having $\mathrm{MIC}_{\mathrm{EC}}$ values $0.42,0.45$ and 0.47 $\mu \mathrm{M} / \mathrm{ml}$, respectively. Compound $\mathbf{8}$ was found to be moderately active against $S$. aureus having $\mathrm{MIC}_{\mathrm{SA}}$ value $0.47 \mu \mathrm{M} / \mathrm{ml}$. In case of antifungal activity against $C$. albicans, compounds 6 was found to be moderately active having $\mathrm{MIC}_{\mathrm{CA}}$ value 0.37 $\mu \mathrm{M} / \mathrm{ml}$ and also compounds $\mathbf{4}$ and $\mathbf{9}$ were found to have promising activity, having $\mathrm{MIC}_{\mathrm{CA}}$ values 0.43 and 0.45 $\mu \mathrm{M} / \mathrm{ml}$.

\section{In vitro antioxidant activity}

Free radical scavenging activity of synthesized compounds (114) against stable free radical DPPH method was determined spectrophotometrically. When DPPH reacts with an antioxidant compound, which can donate hydrogen, it gets converted to reduced form (diphenylpicrylhydrazine). Fifty milliliters of various concentrations $(25,50,75$, and 100) $\mu \mathrm{g} / \mathrm{ml}$ of the compounds dissolved in methanol was added to $5 \mathrm{~mL}$ of a $0.004 \%$ methanol solution of DPPH. After a 30 min incubation period at room temperature, the absorbance was read against a blank at $517 \mathrm{~nm}$. [9, 10] The relative percent of DPPH scavenging activity was calculated according to the following equation:

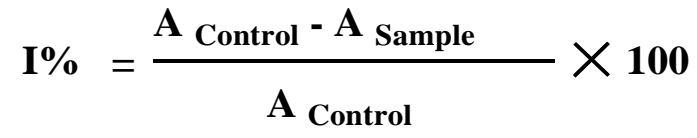

Where,

$\mathbf{A}_{\text {control }}$ is the absorbance of the control reaction.

$\mathbf{A}_{\text {sample }}$ is the absorbance of the test compound.

Compounds concentration providing 50\% inhibition $\left(\mathrm{IC}_{50}\right)$ was calculated from the graph plotted as inhibition percentage against compound concentration (Figures 1, 2 and 3). Tests were carried out in triplicate using ascorbic acid as a positive control. Standard curve is plotted for different concentration of ascorbic acid. From the results of antioxidant activity it was observed that compounds $\mathbf{1}, \mathbf{3}$, and $\mathbf{6}$ have $\mathrm{IC}_{50}$ value 49.38 , 49.48 and $49.75 \mu \mathrm{g} / \mathrm{ml}$, comparable to that of positive control $(49.11 \mu \mathrm{g} / \mathrm{ml})$. 
Indo Global Journal of Pharmaceutical Sciences, 2018; 8(1): 12-20

Table 3: Minimum Inhibitory Concentration (MIC) in $(\mu \mathrm{M} / \mathrm{ml})$ of 3, 4, 5-trihydroxy benzoic acid derivatives against bacterial and fungal Strains.

\begin{tabular}{|c|c|c|c|c|c|}
\hline Compd. & MIC $_{(\mathbf{E C})}$ & $\mathbf{M I C}_{(\mathbf{B S})}$ & $\mathbf{M I C}_{(\mathrm{SA})}$ & MIC $_{(\mathrm{CA})}$ & MIC $_{(\mathrm{AN})}$ \\
\hline $\mathbf{1}$ & $\mathbf{0 . 4 2}$ & $\mathbf{0 . 4 2}$ & $\mathbf{0 . 4 2}$ & $\mathbf{0 . 1 0}$ & $\mathbf{0 . 1 0}$ \\
\hline $\mathbf{2}$ & 1.02 & 1.02 & 1.02 & 0.51 & 1.02 \\
\hline $\mathbf{3}$ & $\mathbf{0 . 4 5}$ & 0.89 & 0.89 & 0.89 & 0.89 \\
\hline $\mathbf{4}$ & 0.86 & 0.86 & 0.86 & $\mathbf{0 . 4 3}$ & 0.86 \\
\hline $\mathbf{5}$ & 0.86 & 0.86 & 0.86 & 0.86 & 0.86 \\
\hline $\mathbf{6}$ & $\mathbf{0 . 3 7}$ & 0.75 & 0.75 & $\mathbf{0 . 3 7}$ & 0.75 \\
\hline $\mathbf{7}$ & 0.89 & 0.89 & 0.89 & 0.89 & 0.89 \\
\hline $\mathbf{8}$ & 0.95 & 0.95 & $\mathbf{0 . 4 7}$ & 0.95 & 0.95 \\
\hline $\mathbf{9}$ & 0.91 & 0.91 & 0.91 & $\mathbf{0 . 4 5}$ & 0.91 \\
\hline $\mathbf{1 0}$ & 0.91 & 0.91 & 0.91 & 0.91 & 0.91 \\
\hline $\mathbf{1 1}$ & 0.91 & 0.91 & 0.91 & 0.91 & 0.91 \\
\hline $\mathbf{1 2}$ & 0.91 & 0.91 & 0.91 & 0.91 & 0.91 \\
\hline $\mathbf{1 3}$ & $\mathbf{0 . 4 7}$ & 0.95 & 0.95 & 0.95 & 0.95 \\
\hline $\mathbf{1 4}$ & 0.91 & 0.91 & 0.91 & 0.91 & 0.91 \\
\hline Std & $0.47^{\mathrm{a}}$ & $0.47^{\mathrm{a}}$ & $0.47^{\mathrm{a}}$ & $0.50^{\mathrm{b}}$ & $0.50^{\mathrm{b}}$ \\
\hline
\end{tabular}

${ }^{\mathrm{a}}$ Norfloxacin, ${ }^{\mathrm{b}}$ Fluconazole, $\mathrm{EC}=E$. coli, $\mathrm{BS}=$ B. subtilis, $\mathrm{SA}=$ S. aureus, $\mathrm{CA}=C$. albicans, $\mathrm{AN}=A$. niger

Table 4: Percentage Inhibition and $\mathrm{IC}_{50}$ value of synthesized compounds (1-14)

\begin{tabular}{|c|c|c|c|c|c|}
\hline Compd. & $\mathbf{2 5}$ & $\mathbf{5 0}$ & $\mathbf{7 5}$ & $\mathbf{1 0 0}$ & $\mathbf{I C}_{\mathbf{5 0}}$ \\
\hline $\mathbf{1 .}$ & 38.19 & 47.35 & 65.89 & 77.89 & 49.38 \\
\hline $\mathbf{2 .}$ & 28.19 & 50.00 & 65.89 & 79.12 & 54.01 \\
\hline $\mathbf{3 .}$ & 45.02 & 47.61 & 56.66 & 68.09 & 49.48 \\
\hline $\mathbf{4 .}$ & 39.42 & 45.87 & 61.78 & 77.32 & 51.08 \\
\hline $\mathbf{5 .}$ & 39.18 & 45.35 & 64.89 & 77.89 & 50.33 \\
\hline $\mathbf{6 .}$ & 32.40 & 51.23 & 67.89 & 90.76 & 49.75 \\
\hline $\mathbf{7 .}$ & 44.88 & 47.14 & 52.85 & 59.52 & 57.19 \\
\hline $\mathbf{8 .}$ & 42.65 & 47.14 & 51.90 & 89.52 & 52.64 \\
\hline $\mathbf{9 .}$ & 36.89 & 42.90 & 47.12 & 51.90 & 82.24 \\
\hline $\mathbf{1 0 .}$ & 41.70 & 46.23 & 50.49 & 57.78 & 57.78 \\
\hline $\mathbf{1 1 .}$ & 45.76 & 49.10 & 53.87 & 59.12 & 51.66 \\
\hline $\mathbf{1 2 .}$ & 29.03 & 37.14 & 61.78 & 73.12 & 62.08 \\
\hline $\mathbf{1 3 .}$ & 37.91 & 42.38 & 57.61 & 71.42 & 57.67 \\
\hline $\mathbf{1 4 .}$ & 41.23 & 45.23 & 50.47 & 54.76 & 73.80 \\
\hline Ascorbic acid* & $\mathbf{2 8 . 1 8}$ & $\mathbf{5 0 . 6 7}$ & $\mathbf{7 5 . 9 8}$ & $\mathbf{9 1 . 8 9}$ & $\mathbf{4 9 . 1 1}$ \\
\hline
\end{tabular}

*Positive control

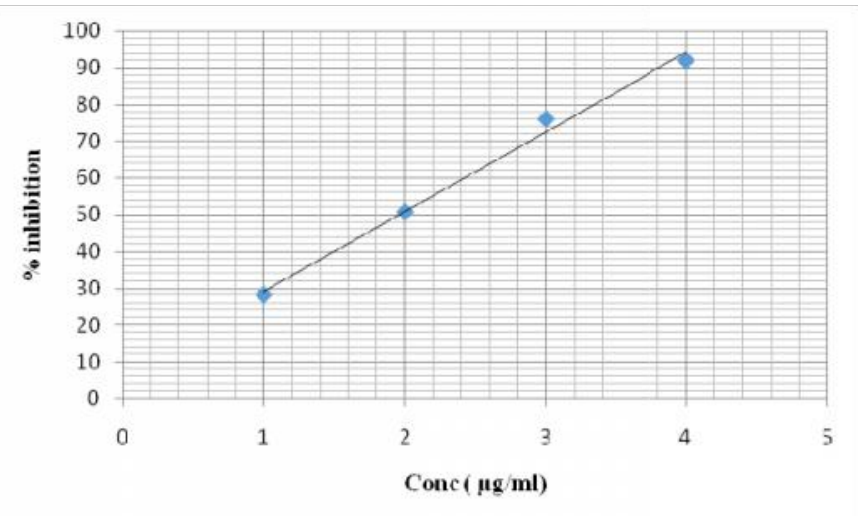

Figure 1: Standarad curve of ascorbic acid

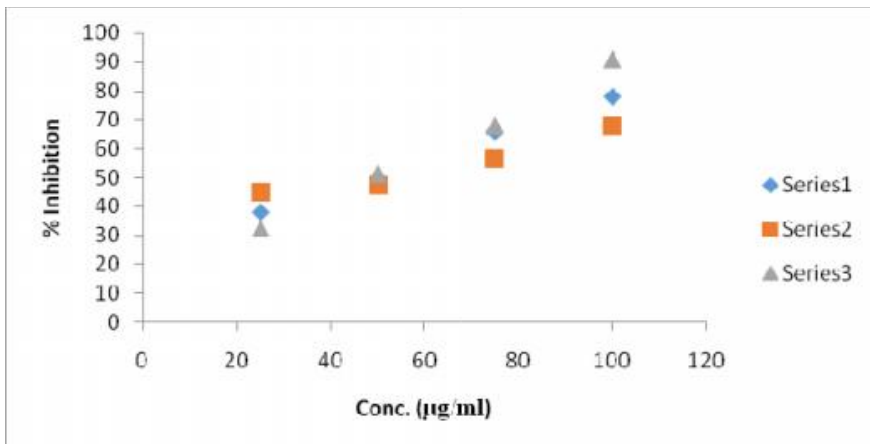

Figure 2: Percentage Inhibition value of active antioxidant compounds $1,3,6$. * Series 1 indicate percentage inhibition of compound 1, Series 2 indicate percentage inhibition of compound 3, and Series 3 indicate percentage inhibition of compound 6 . 
Indo Global Journal of Pharmaceutical Sciences, 2018; 8(1): 12-20

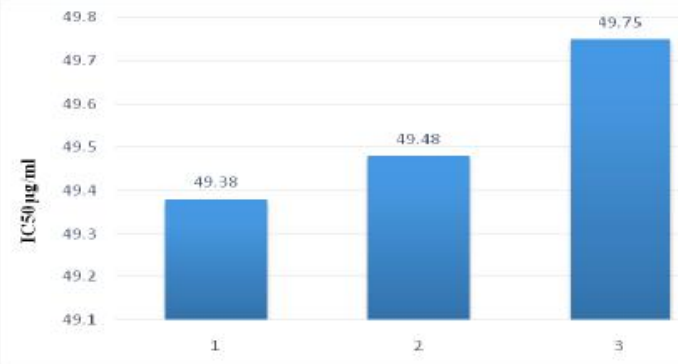

Figure 3: $\mathbf{I C}_{\mathbf{5 0}}$ values of most potent compounds. $* 1,2$ and 3 indicates most potent compounds 1,3 and 6

\section{Urease inhibition assay}

Urease inhibitory activity for each synthesized compounds (1-14) was determined using Jack Bean Urease by Indophenol method. At first, $40 \mathrm{~L}$ buffer (100 mM urea, $1 \mathrm{mM}$ EDTA, $0.01 \mathrm{M}$ $\left.\mathrm{K}_{2} \mathrm{HPO}_{4}, 0.01 \mathrm{M} \mathrm{LiCl}_{2}, \mathrm{pH} 8.2\right), 10 \mathrm{~L}$ of test compound and 10 L of enzyme $(5 \mathrm{U} / \mathrm{mL})$ solution were incubated at $37{ }^{0} \mathrm{C}$ for 10 min. In addition, solutions of $40 \mathrm{~L}$ of alkali reagent $(0.5 \%, \mathrm{w} / \mathrm{v}$ $\mathrm{NaOH}, 0.1 \%$ active chloride $\mathrm{NaOCl}$ ) and $40 \mathrm{~L}$ of phenol reagent ( $1 \%$, w/v phenol, $0.005 \%$, w/v sodium nitroprusside) were introduced to each well. Experiment study was determined at 625 $\mathrm{nm}$ in UV spectrophotometer and thiourea was used as standard inhibitor. The percentage inhibition was calculated using the following equation:

$$
I \%=\frac{A_{\text {Control }}-A_{\text {Sample }}}{A_{\text {Control }}} \times 100
$$

Where,

$\mathbf{A}_{\text {control }}$ is the absorbance of the positive control.

$\mathbf{A}_{\text {sample }}$ is the absorbance of the test compound.

From the results of urease inhibitory activity it was observed that all of the synthesized compounds showed good activity. Compound 13 was found to be most active among all the synthesized compounds having $\mathrm{IC}_{50}$ value $0.07 \mu \mathrm{M} / \mathrm{ml}$ when compared with positive control thiourea $(0.61 \mu \mathrm{M} / \mathrm{ml})$. Compound 1 was found to be moderately active having $\mathrm{IC}_{50}$ value of $0.08 \mu \mathrm{M} / \mathrm{ml}$.

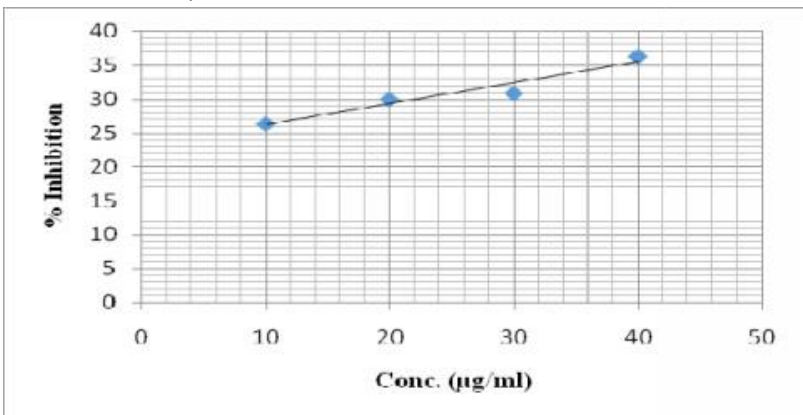

Figure 4: Standard curve of Thiourea

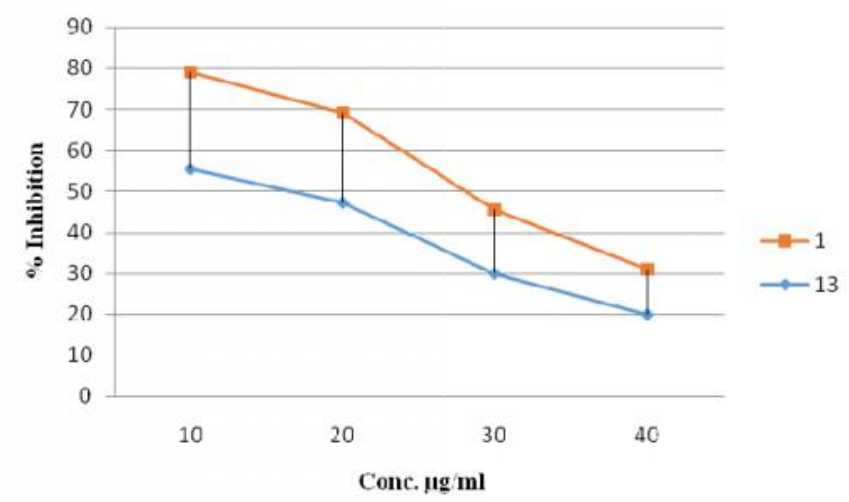

Figure 5: Percentage Inhibition value of active Urease potential compounds 13 and 1

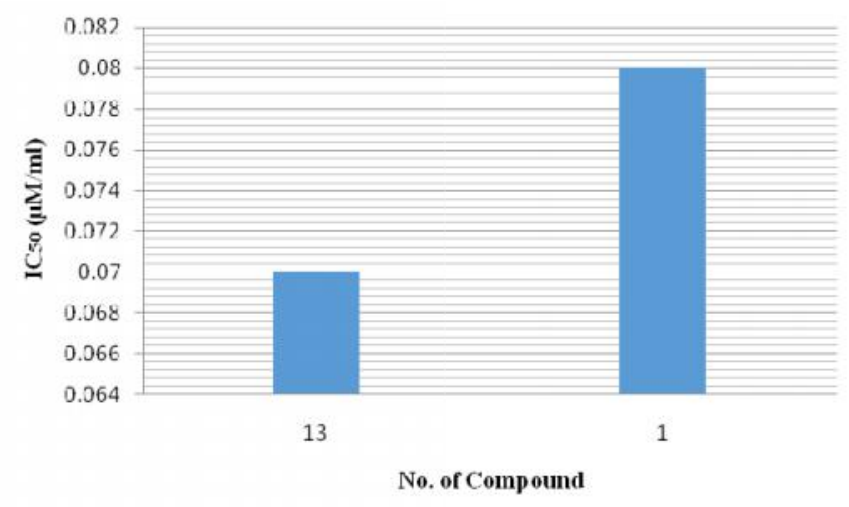

Figure 6: $\mathrm{IC}_{50}$ values of most potent compounds

\section{STRUCTURE ACTIVITY RELATIONSHIP} $\underline{(\text { SAR })}$

From the aforementioned biological activities of the 3, 4, 5trihydroxy benzoic acid derivatives, the following structure activity relationships can be derived (Figure 7):

* Substitution with 8-hydroxy quinoline (heteroaryl group) at $\mathrm{R}_{1}$ position, as indicated in compound quinolin-8-yl 3,4,5trihydroxybenzoate (1) shows most potent antimicrobial, antioxidant activity and also possesses good urease inhibitory potential.

* In case of alkoxy group (electron donating group) substitution at phenyl ring attached to hydroxybenzamide ring, as present in compound 3,4,5-trihydroxy-N-(2 methoxyphenyl) benzamide (9) increases antifungal activity against $C$. albicans.

* Substitution of para flouro group in compound N-(4fluorophenyl)-3,4,5-trihydroxybenzamide (13) increases urease inhibitory activity.

From these results we may conclude that different structural requirements are required for a compound to be effective against different targets. 
Indo Global Journal of Pharmaceutical Sciences, 2018; 8(1): 12-20

Table 6: $\mathrm{IC}_{50}$ value of synthesized compounds (1-14)

\begin{tabular}{|c|c|c|c|c|c|}
\hline Compound & $\mathbf{1 0}(\mathrm{g} / \mathrm{ml})$ & $\mathbf{2 0}(\mathrm{g} / \mathrm{ml})$ & $\mathbf{3 0}(\mathrm{g} / \mathrm{ml})$ & $\mathbf{4 0}(\mathrm{g} / \mathrm{ml})$ & $\mathbf{I C}_{\mathbf{5 0}}(\mathrm{M} / \mathrm{ml})$ \\
\hline 1. & 23.63 & 21.81 & 15.45 & 10.90 & $\mathbf{0 . 0 8}$ \\
\hline $\mathbf{2 .}$ & 11.81 & 19.09 & 23.63 & 30.00 & 0.25 \\
\hline $\mathbf{3 .}$ & 11.27 & 16.36 & 23.63 & 24.54 & 0.26 \\
\hline $\mathbf{4 .}$ & 16.36 & 20.90 & 20.90 & 20.90 & 0.23 \\
\hline $\mathbf{5 .}$ & 13.63 & 16.36 & 20.00 & 30.13 & 0.18 \\
\hline $\mathbf{6 .}$ & 14.57 & 17.27 & 30.00 & 32.72 & 0.16 \\
\hline $\mathbf{7 .}$ & 10.90 & 13.63 & 20.00 & 28.18 & 0.20 \\
\hline $\mathbf{8 .}$ & 19.09 & 25.45 & 38.18 & 43.46 & 0.16 \\
\hline $\mathbf{9 .}$ & 11.22 & 12.65 & 13.63 & 23.63 & 0.16 \\
\hline $\mathbf{1 0 .}$ & 21.81 & 25.45 & 30.00 & 38.18 & 0.23 \\
\hline $\mathbf{1 1 .}$ & 19.09 & 28.18 & 30.00 & 40.90 & 0.12 \\
\hline $\mathbf{1 2 .}$ & 10.90 & 20.00 & 30.00 & 38.18 & 0.17 \\
\hline $\mathbf{1 3 .}$ & 55.45 & 47.27 & 30.00 & 20.00 & $\mathbf{0 . 0 7}$ \\
\hline $\mathbf{1 4 .}$ & 11.45 & 16.33 & 20.12 & 35.22 & 0.17 \\
\hline Thiourea* & 26.36 & 30.00 & 30.90 & 36.36 & 0.61 \\
\hline
\end{tabular}

*Positive control

Figure 7: Structure-activity relationship of the 3, 4, 5-trihydroxy benzoic acid derivatives

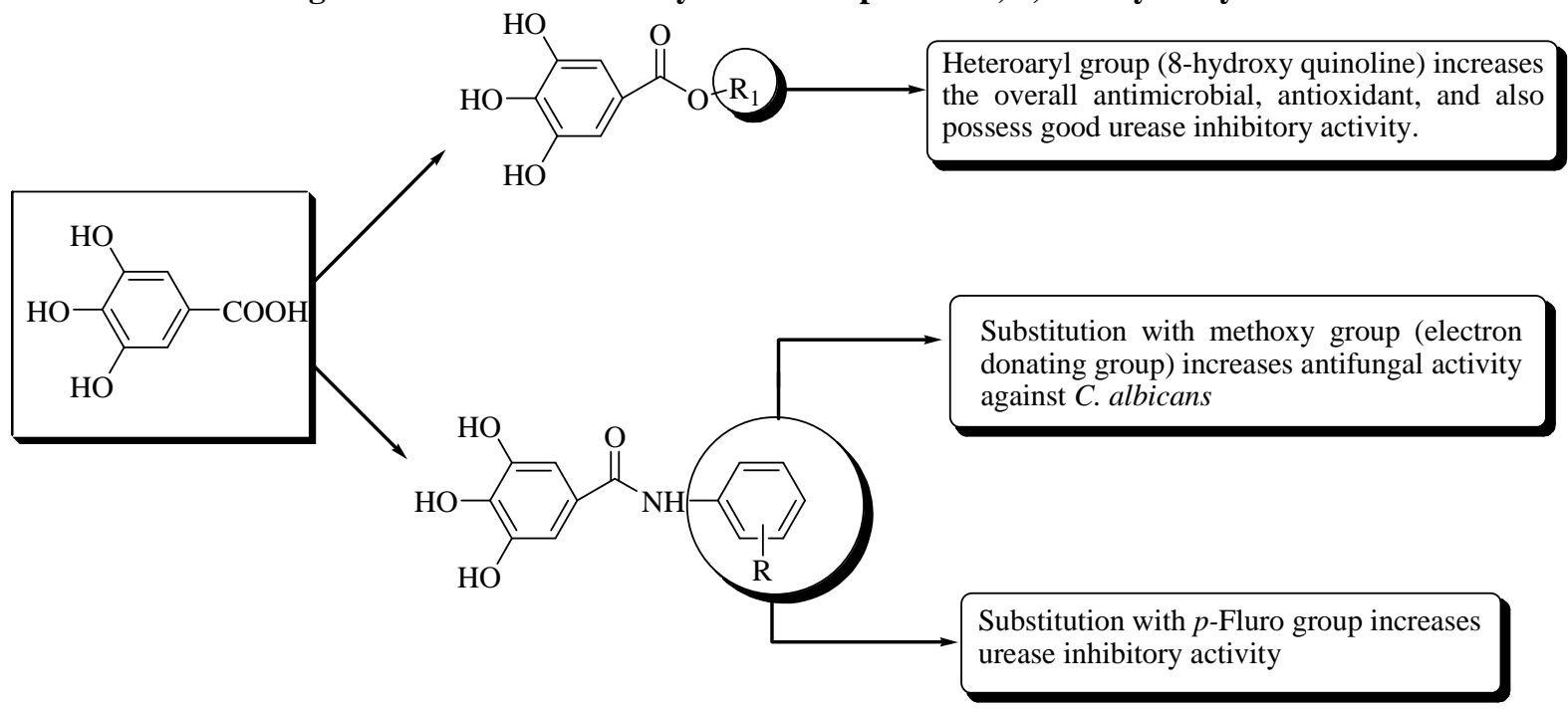

\section{CONCLUSION}

In the present study, a novel series of 3, 4, 5-trihydroxy benzoic acid derivatives (1-14) was synthesized and evaluated in vitro for its antimicrobial activity by the tube dilution method. Results of antimicrobial screening indicated that compound quinolin-8-yl 3,4,5-trihydroxybenzoate (1) was the most active antimicrobial agent $\left(\mathrm{MIC}_{\mathrm{CA}}=\mathrm{MIC}_{\mathrm{AN}}=0.10\right.$ $\mu \mathrm{M} / \mathrm{ml})$, as well as showed good antioxidant potential $\left(\mathrm{IC}_{50}=49.38 \mu \mathrm{g} / \mathrm{ml}\right)$. The synthesized derivatives $(\mathbf{1 - 1 4})$ were also evaluated in vitro for their urease inhibitory activities; most of the investigated compounds were potent inhibitors of Jack Bean Urease by Indophenol method. The compounds $\mathbf{1 3}$ and $\mathbf{1}$ were found to be more potent urease inhibitors than the standard thiourea, depicting the $\mathrm{IC}_{50}$ values of 0.07 and 0.08 $\mu \mathrm{M} / \mathrm{ml}$, respectively $\left(\mathrm{IC}_{50}\right.$ of thiourea $\left.=0.61 \mu \mathrm{M} / \mathrm{ml}\right)$.

\section{REFERENCES}

[1] I. M. Gould. The epidemiology of antibiotic resistance. Int. J. Antimicrob. Agents, 2008, 32: S2-S9.

[2] K. Sieradzki, R. B. Roberts, S. W. Haber, et al. The development of vancomycin resistance in a patient with methicillin-resistant Staphylococcus aureus infection. New Engl. J. Med., 1999, 340: 517-523.

[3] T. Nitta, T. Arai, H. Takamatsu, et al. Antibacterial activity of extracts prepared from tropical and subtropical plants on methicillinresistant Staphylococcus aureus. J. Health Sci., 2002, 48: 273-276. 
Indo Global Journal of Pharmaceutical Sciences, 2018; 8(1): 12-20

[4] D. P. Kontoyiannis, E. Mantadakis, G. Samonis. Systemic mycoses in the immunocompromised host: an update in antifungal therapy. J. Hosp. Infect., 2003, 53: 243-258.

[5] M. Hanif, K. Shoaib, M. Saleem, N. Hasan Rama, et al. Synthesis, urease inhibition, antioxidant, antibacterial, and molecular docking studies of 1, 3, 4-oxadiazole derivatives. ISRN Pharmacol., 2012: 2012.

[6] H. L. Mobley, M. D. Island, R. P. Hausinger. Molecular biology of microbial ureases. Microbiol. Rev., 1995, 59: 451-480.

[7] S. Vassiliou, A. Grabowiecka, P. Kosikowska, et al. Design, synthesis, and evaluation of novel organophosphorus inhibitors of bacterial ureases. J. Med. Chem., 2008, 51: 5736-5744.

[8] Y. P. Xu, J. Qin, S. M. Sun, et al. Synthesis, crystal structures, molecular docking and urease inhibitory activity of nickel (II) complexes with 3-pyridinyl-4-amino-5-mercapto-1, 2, 4triazole. Inorg. Chimica. Acta., 2014, 423: 469-476.

[9] A. Saeed, M. S. Khan, H. Rafique, et al. Design, synthesis, molecular docking studies and in vitro screening of ethyl 4-(3benzoylthioureido) benzoates as urease inhibitors. Bioorg. Chem., 2014, 52: 1-7.

[10] N. C. Ha, S. T. Oh, J. Y. Sung, et al. Supramolecular assembly and acid resistance of Helicobacter pylori urease. Nat. Struct. Mol. Biol., 2001, 8: 505-509.

[11] E. Jabri, M. B. Carr, R. P. Hausinger, et al. The crystal structure of urease from Klebsiella aerogenes. Sci., 1995, 268: 998-1004.

[12] X. D. Yu, R. B. Zheng, J. H. Xie, et al. Biological evaluation and molecular docking of baicalin and scutellarin as Helicobacter pylori urease inhibitors. J. Ethnopharmacol., 2015, 162: 69-78.

Indo Global Journal of Pharmaceutical Sciences( ISSN 22491023 ; UGC Journal No.: 44477; CODEN- IGJPAI; NLM ID: 101610675) indexed and abstracted in EMBASE(Elsevier), UGC Journal List, National Library of Medicine (NLM) Catalog, Elsevier( EMBASE), ResearchGate, Publons, CAS (ACS), Index Copernicus, Google Scholar and many more. For further details, visit http://iglobaljournal.com 